\title{
Plataformas educativas de nivel básico usadas en homeschooling en México
}

\author{
Basic level educational platforms used in homeschooling in Mexico \\ Liliana Ramírez-Vera ${ }^{a}$, M. Guadalupe Veytia ${ }^{b}$, Rebecca English ${ }^{c}$
}

\begin{abstract}
:
Distance education is a form of education that has increased considerably since the middle of the last century not only in Primary and Secondary Education but also in Higher Education. The use of Information and Communication Technologies is one of the main factors for its development since it has allowed distances to be shortened and communication has been established in both synchronous and asynchronous spaces. The aim of this work is to characterize this educational practice in Mexico. The study is directed towards a qualitative analysis of Virtual Learning Environments (VLE) through the management of Educational Platforms in the families that use them at the Primary Education level. Among the main findings was the management of four platforms: MEVyT Online, educazion.net, aprendeconalas.com and matihomeschool.com. It concluded that the mediation work carried out by parents in this process plays a fundamental role so that students acquire autonomy and identify their learning style and rhythm. In addition, they learn to use different technological resources.
\end{abstract}

\section{Keywords:}

Homeschooling, Basic level, Mexico, Educational Plataforms, Technology

\section{Resumen:}

La Educación a Distancia es una modalidad educativa que se ha incrementado considerablemente a mediados del siglo pasado tant o en Educación Básica como en Media Superior, Superior y Posgrado, siendo el empleo de las Tecnologías de la Información y la Comunicación uno de los principales factores para su desarrollo, ya que ha permitido acortar distancias, y establecer comunicación tanto en espacios sincrónicos como asincrónicos. El objetivo de este trabajo es caracterizar esta práctica educativa en México, dirigiendo el estudio hacia una reflexión de Entornos Virtuales de Aprendizaje (EVA), mediante el manejo de Plataformas Educativas en las familias que las emplean en el nivel de Educación Básica Primaria. Dentro de los principales hallazgos se encontraron el manejo de cuatro plataformas: MEVyT en línea, educazion.net, aprendeconalas.com, matihomeschool.com. Se concluye que el trabajo de mediación que realizan los padres de familia en este proceso desempeña un papel fundamental para que los estudiantes adquieran autonomía, identifiquen su estilo y ritmo de aprendizaje, además de que aprendan a aprender utilizando diferentes recursos tecnológicos.

\section{Palabras Clave:}

Homeschooling, Nivel Básico, México, Plataformas Educativas, Tecnología

\footnotetext{
a Autor de Correspondencia, Universidad Autónoma del Estado de Hidalgo, México, https://orcid.org/0000-0003-1451-8487, Email: lili.ramirez0610@gmail.com

b Universidad Autónoma del Estado de Hidalgo, México, https://orcid.org/0000-0002-1395-1644, Email: maria_veytia@uaeh.edu.mx c Universidad Tecnológica de Queensland, Australia, https://orcid.org/0000-0002-4039-6105, Email: r.english@qut.edu.au
} 


\section{Introducción}

El Homeschooling es el término en inglés para la Educación en Casa, cuando los niños entre 6 y 12 años de edad reciben la instrucción académica en el hogar, donde los padres deciden brindar a sus hijos edu cación de acuerdo a sus convicciones y a lo que consideran más óptimo para ellos o de acuerdo a los intereses, capacidades y estilos de aprendizaje de cada niño. Esta modalidad de educación, integra la instrucción de los hijos tanto en conocimientos conceptuales como procedimentales y actitudinales, al ritmo de aprendizaje y las necesidades de cada menor (Hoeneisen, 2014).

Para este documento, se refiere al fenómeno como Educación en casa o bien Homeschooling. Ambos términos del fenómeno educativo refieren que es un método de enseñanza alternativo al escolarizado, en el que cada familia decide la forma de aprendizaje de sus hijos, así como el tiempo y ritmo al que deben hacerlo. Siendo esta alternativa o modalidad educativa llevada con un currículum definido, ya sea mediante Plataformas Educativas o mediante libros que los padres proporcionan a sus hijos para su formación académica (Homeschooling) o sin un currículum, solo dan a sus hijos los materiales de su interés (Unschooling) o incluso una mezcla de apoyo a las instituciones educativas con Plataformas Educativas (Flexischooling).

\section{Investigaciones en México respecto del Homeschooling}

En México no se conoce el número de familias que educan en casa, ya que al no haber una reglamentación clara, las familias simplemente no tienen ningún registro oficial que permita contabilizarlas y con ello conocer cifras exactas. No obstante, la información de redes sociales, publicaciones e investigaciones como la de Abraham (2016) de la Universidad Nacional Autónoma de México (UNAM) y la de Sánchez (2016) de Universidad Pedagógica Nacional (UPN), de la última década, indican que existe una población significativa con rasgos diversos que practican la educación sin enviar a los niños a la escuela.

Alvarado y Cerón (2015) analizan al Homeschooling desde el ángulo del currículum estandarizado y mu ch as veces obsoleto que se aplica en las escuelas, el cual no favorece a todos los niños, desde sus características, necesidades y estilos de aprendizaje. El currículum funciona como un dispositivo detalladamente formulado para adoctrinar a las masas, formando gente dócil de manejar y controlar, educada bajo una sola alternativa: la obligada asistencia a las escuelas, para recibir educación. Concluyen que una de las grandes fortalezas del modelo de escuela en casa, es que los padres tienen la libertad de decidir el currículum para educar a sus hijos, evitando así, exponerlos a las ideologías homogeneizantes que el Estado pretende imponer con el apoyo de un cu rículum oficial estandarizado.

Abraham (2016) lleva a cabo un análisis crítico de cómo en México existen diversos problemas en el sistema educativo en lo que a calidad, cobertura e infraestructura se refiere. Describe situaciones que existen específicamente dentro de las aulas, -como el acoso escolar. Por otro lado, la Organización para la Cooperación y el Desarrollo Económicos (OCDE) publicó en 2015, que México tiene el primer lugar en bullying; y que un gran número de alumnos en los salones impide atención suficiente. Ante estas problemáticas, algunos padres buscan soluciones que les permitan a sus hijos desarrollarse de manera integral y armónica, que cumpla con los estándares deseados desde un ambiente seguro. Por tanto, la OCDE reconoce a la Educación en casa como una posible solución ante la problemática detectada. Dentro de los principales hallazgos en este estudio se encuentra que la Secretaría de Educación Pública (SEP) no reconoce esta opción de educación en México, y que existe un vacío legal, por lo que el Homeschooling no está prohibido, pero tampoco está legalmente constituido y fundamentado para su práctica, y no hay quien regule sus procesos.

Alvarado, Montemayor y Rosales (2018) abordan el tema de la Educación en casa en México, a través de las percepciones de tres familias de religión cristiana, de clase media de la ciudad de Monterrey, México, describiendo el contexto sociocultural de estas familias que optaron por el modelo de Educación en Casa para la formación Básica (Primaria y Secundaria) y Media Superior (Preparatoria) de sus hijos, a fin de influir en su desarrollo con relación al ámbito social y académico. En su estudio, identifican que la educación en el hogar se ha convertido en una modalidad cada vez más aprovechada por las familias, la cual es practicada por cerca de 84,000 familias en el mundo. En Monterrey, más de 500 familias están optando por una alternativa pedagógica distinta a la tradicional. Sin embargo, predomina la postura desinformada respecto al tema. De acuerdo con los datos recopilados en esta investigación, las familias tuvieron como preocupación principal el dar una educación de mayor calidad a sus hijos, se coincide en la educación personalizada y al ritmo individual de cada niño. En las familias entrevistadas resulta ser la madre el pilar fundamental para que la educación en casa funcione.

El fenómeno de la Educación en Casa ha crecido en México en las últimas décadas, por diversas razones. De acuerdo con Sánchez (2016), los principales motivos son: pedagógicos, éticos o morales, sociales $\mathrm{y} / \mathrm{o}$ religiosos. El crecimiento se desarrolla desde un contexto 
de desconocimiento de los sustentos teóricos, así como del alcance académico y sociocultural que se gen era en esta práctica.

Podemos considerar que de acuerdo con los referentes planteados tampoco existe mucha investigación al respecto, por lo que resulta de gran importancia dar inicio a mayores estudios que permitan dar cuenta de este fenómeno y de qué manera podría contribuir con la calidad de la educación y el aminoramiento del rezago educativo en México.

\section{Plataformas Educativas en México}

En este contexto de cambios socioculturales y a partir del uso creciente de los Entornos Virtuales de Aprendizaje (EVA), es que se pretende analizar y describir, bajo el modelo del Homeschooling, cómo los contextos virtuales influyen en el niño, esto a partir del empleo de nuevas herramientas para favorecer el proceso de aprendizaje que realizan. Además de analizar cómo es que los padres, como tutores o supervisores responsables de este proceso educativo, junto con una sistematización de contenidos en Plataformas Educativas, logran que el niño construya y aprenda conocimientos, y pueda generar una conciencia social de su entorno como ciudadano global. La modalidad, objeto de estudio de esta investigación es el aprendizaje auto-dirigido mediado por las Tecnologías de la Información y Comunicación (TIC), que ha permitido un crecimiento tanto del fenómeno del Homeschooling como del conocimiento de los EVA, mediante Plataformas Educativas, utilizados por las familias que educan en casa.

De acuerdo con Luis Gutiérrez, actualmente los programas educativos hacen uso de las tecnologías como una herramienta digital fundamental en las experiencias de aprendizaje; afirma que "de esta forma, se supera la concepción tradicional de que sólo el aprendizaje emerge en los espacios físicos y tiempos delimitados por las instituciones educativas" (Gutiérrez, 2012: 1).

De la misma manera, en la actualidad existe una creciente dinámica social en EVA, en la que es innegable la participación de todos los sectores de la población en las TIC. La creciente demanda educativa en contextos virtuales hace necesario justificar y fundamentar conceptual y teóricamente sus procesos en la Educación Básica. Es por ello, que se pretende profundizar en el estudio del Homeschooling, como una modalidad que crece en una era donde las tecnologías aumentan y transforman el desarrollo educativo y sociocultural de los niños con nuevos instrumentos, agentes y contextos (Pineda, 2016).

Al respecto, Coll y Monereo (2008) mencionan que en tre los Entornos Virtuales de Enseñanza y Aprendizaje se encuentran los sistemas expertos de emulación del aprendizaje colaborativo, plataformas y estándares elearning y comunidades virtuales, y que éstos tienen mayor presencia tanto en los proyectos educativos más innovadores como en los trabajos de investigación aplicada.

A partir de la búsqueda realizada, se encontró que en México existen cuatro Plataformas Educativas usadas para Educación Básica por niños que son educados por sus padres o tutores en casa, y que se emplean para este tipo de formación.

La primera de estas plataformas es el MEVyT en línea de la SEP, siguiendo con algunas Plataformas Educativas privadas mexicanas, extranjeras o mexicano-extranjeras, que son usadas por familias que hacen Homechooling, tales como: MEVyT en línea, educazion.net, aprendeconalas.com y matihomeschool.com, cuyos datos particulares, como sus respectivos links, se presentan en la tabla 1.

Tabla 1 Plataformas Educativas usadas en Homeschooling en México.

\begin{tabular}{|c|c|c|}
\hline $\begin{array}{l}\text { Nombre de la } \\
\text { Plataforma } \\
\text { Educativa }\end{array}$ & Link & $\begin{array}{c}\text { Uso de la } \\
\text { educación en } \\
\text { casa }\end{array}$ \\
\hline $\begin{array}{l}\text { MEVyTen } \\
\text { línea }\end{array}$ & $\begin{array}{l}\text { http://mevytenlinea.inea.go } \\
\text { b.mx/inicio/index.html }\end{array}$ & $\begin{array}{l}\text { Flexischooling y } \\
\text { Homeschooling }\end{array}$ \\
\hline educazion.net & https://educazion.net/info/ & Homeschooling \\
\hline $\begin{array}{l}\text { Aprendeconal } \\
\text { as.com }\end{array}$ & $\begin{array}{l}\text { https://www.aprendeconalas. } \\
\text { com/ }\end{array}$ & Homeschooling \\
\hline $\begin{array}{l}\text { Matihomesch } \\
\text { ool.com }\end{array}$ & $\begin{array}{l}\text { http://www. matihomeschool. } \\
\text { com/ }\end{array}$ & $\begin{array}{l}\text { Flexischooling y } \\
\text { Homeschooling }\end{array}$ \\
\hline
\end{tabular}

Fuente: Elaboración propia

Clarenc (2013) menciona que las Plataformas Educativas como Learning Management System (LMS) o Sistema de Gestión del Aprendizaje, consisten en un software instalado generalmente en un servidor web, que se emplea para crear, aprobar, administrar, almacenar, distribuir y gestionar las actividades de formación virtual, y que pueden usarse como complemento de clases presenciales o para el aprendizaje a distancia. Estos sistemas se centran en gestionar contenidos creados por una gran variedad de fuentes diferentes.

Una Plataforma Educativa se considera un ambiente, donde el aprendizaje se convierte en una verdadera experiencia, integrando materiales didácticos y herramientas de comunicación, colaboración y gestión educativa, que son susceptibles de ser analizados para conocer la efectividad de estos entornos virtuales, tan usados en la educación actualmente (Clarenc, 2013).

Debido al uso de las TIC, los alumnos con posibilidad de tener acceso a una computadora, internet y por medio de alguna Plataforma Educativa pueden ir realizando y comprobando sus avances, en cuanto a sus estudios académicos de la Educación Básica Primaria, con un carácter innovador, ya que los ambientes de aprendizaje 
van cambiando y los contextos se van adaptando a las nuevas modalidades de procesos de enseñanza y aprendizaje.

En el 2017, el Instituto Nacional para la Educación de Adultos (INEA) publicó, datos importantes respecto a la introducción del programa a distancia llamado "MEVyT en línea", el cual se fusionó con el programa "MEVyT 1014", desarrollado en el 2014, por medio del cual los niños que realizan Homeschooling son certificados en México. En los datos reportados se puede observar un alto crecimiento, ya que de 403 alumnos, que se tenían sin el programa de certificación, un año después de in troducir el Mevyt 10-14, en 2015 la cantidad de alumnos se incrementó a 23,250. Con estas cifras, podemos identificar el aumento en la cantidad de los niños con edades entre los 10 y 14 años de edad que se certifican en educación primaria, sin haber asistido a la escuela.

Para ampliar el panorama respecto a estas estadísticas, se realizó una entrevista a la Coordinadora de Formación y enseñanza del Instituto Hidalguense de Educación para los Adultos (IHEA), quien respondió algunas preguntas acerca de la modalidad del MEVyT en línea y el MEVyt 10-14. Este programa es una modalidad en línea para certificar la Educación Básica Primaria y Secundaria, incluye la versión del 10-14, modalidad en la que los niños de este rango de edad, que no van a la escuela, pueden certificar su educación primaria por medio de 12 módulos en línea. De acuerdo con la coordinadora, no se encuentran muchos niños que sean educados en casa en esta certificación. Sin embargo, anualmente, el número de niños que certifican puede variar, siendo entre 15 y 20 niños en la capital del Estado de Hidalgo que reciben instrucción académica en el hogar por parte de sus padres o tutores, y los demás son niños que no van a la escuela porque se encuentran reclusos en Consejos Tutelares 0 en casas de niños del DIF municipal (Sistema para el Desarrollo Integral de la Familia del Estado de Hidalgo).

La coordinadora comentó que no existe ningún problema legal con los padres que llevan a certificar a sus hijos en esta modalidad, el sistema es gratuito y obtienen la certificación en un periodo que va de los 6 a los 10 meses. Una vez cumplidos los 15 años, los adolescentes pueden certificar, con un solo examen, su educación Secundaria, lo que corresponde a la edad en la que concluyen sus estudios de secundaria en una modalidad presencial. De la misma manera, expresó que a veces se usa el MEVyT en línea para los niños superdotados, ya que quieren terminar rápido la primaria, sin embargo, para certificar secundaria tendrían que esperar a cumplir los 15 años.

Aunque el crecimiento del Homeschooling en México no es medible ni regulado aún, como ya se había mencionado, la existencia de programas para la certificación oficial de niños que no asisten a las instituciones escolares tradicionales, puede dar una idea del comportamiento de los datos al respecto, y aunque existen otros métodos de certificación, como la revalidación de certificados expedidos en el extranjero, opción que toman las demás Plataformas Educativas mencionadas en este trabajo que son: educazion.net, aprendeconalas.com y Matihomeschool.com; los datos que arroja el programa de INEA sigue siendo el más conocido, además de que es el programa a nivel nacional, donde los niños de todos los estados de la República Mexicana acuden a esta certificación, ahora con todos los recursos en línea; incluso, mediante el mismo programa en plataforma educativa, se puede acceder a los módulos y contenidos que permiten la validez oficial del aprendizaje de niños que son educados en casa.

\section{Factores del contexto sociocultural}

De acuerdo con Coll y Monereo (2008), desde hace algunas décadas se van dando cambios sociales, económicos, políticos y culturales que dan origen a una reorganización social, identificada como Sociedad de la Información, en la que en gran medida se han desarrollado las TIC, dando origen a nuevos paradigmas tecnológicos asociados a cambios sociales, culturales y económicos profundos.

De acuerdo con los retos de la globalización, los contextos tienen que ir adaptándose a nuevos métodos de comunicación y desarrollo. Lo que lleva a una sociedad que termina siendo asistida por una computadora o equipo tecnológico en casi todos los aspectos de la vida y sobre todo, en el educativo. En el ámbito educativo, la educación asistida por la tecnología se le conoce como e-learning. De esta manera se comprende que los cambios en la tecnología y la transformación de herramientas, sean los que medien las actividades y los sujetos que las realizan.

De igual forma el acceso universal y casi ilimitado a los textos y la comunicación, como son el correo electrónico, las redes sociales, chats, foros, SMS transforman a los sujetos en cuanto a sus prácticas de socialización. Esto es porque el fenómeno Internet conforma además un nuevo y complejo espacio global para la acción social, que se extiende al aprendizaje y la acción educativa (Castells, 2000).

De acuerdo con Shayo, Olfman, Iriberri \& Igbaria (2007), esto ha ido reconfigurando las formas de la sociedad, Io mismo para el trabajo, que para los servicios, incluso para las relaciones sociales significativas. Lo que ha dado origen a formas sociales virtuales en aspectos corporativos, alfabetización digital a la población, nuevas infraestructuras y por lo tanto, a una política de apoyo a la virtualidad. 
En este mismo sentido, se puede identificar que el desarrollo de la Sociedad de la Información y de las TIC va produciendo cambios en las vidas de las personas y en los grupos sociales. De acuerdo con Coll y Monereo (2008: 54), el desarrollo, puede ser identificado como la "participación cambiante en las actividades socioculturales de la propia comunidad, la cual también se halla en proceso de cambio". La tecnología entonces, juega un papel esencial en los procesos de evolución de los entornos sociales, claro esto planteado desde una visión constructivista.

Desde los planteamientos anteriores, resulta relevante distinguir el impacto sociocultural que las tecnologías y en particular los entornos virtuales, han generado en familias que utilizan esta modalidad. Ya que si bien cada sociedad cumple las tareas de socialización de sus miembros, cada sociedad transmite una cultura que, en un momento dado, es compartida por la mayoría de sus miembros, intentando que al final de todo ello, inicialmente arbitrario, se termine aceptando lo aprendido como normal, como bueno o como natural (Mieles \& García 2010). Es así que cada sociedad, utilizando diferentes agentes y procesos más o menos explícitos e intencionales, enseña 0 transmite a las nuevas generaciones una estructura de conocimientos, códigos, representaciones, reglas formales e informales, modelos de comportamiento y de valores, intereses, aspiraciones, cosmovisiones, creencias, mitos.

\section{A manera de conclusión}

Si bien este trabajo ha planteado una serie de reflexiones en torno a lo que significa enseñar en casa, es importante reconocer que los cambios que se suscitan en la actualidad deben conducirnos a la tran sformación de las prácticas educativas tradicionales, puesto que la escuela, como la conocemos hasta ahora, no está dando respuesta a las nuevas necesidades de la sociedad actual. De manera particular, vemos que los alumnos acceden con facilidad a la información a partir del empleo de las Tecnologías de la Información y la Comunicación, sin embargo, en ocasiones, se quedan únicamente con la información sin generar procesos de análisis y reflexión, por lo que algunos padres de familia al ver las necesidades de sus hijos, optan por trabajar esta modalidad de estudio en casa, de tal manera que ellos sean quienes los puedan orientar en proceso, y les ayuden a generar aprendizajes significativos.

A la fecha, en México no existen opciones que den cabida a las nuevas necesidades educativas, ya que se debe esperar a que los niños reúnan la característica de extra-edad (10-14), que a su vez, para el sistema educativo se convierte en rezago, para poder dar opción a un proceso de certificación de la educación básica fuera de sus aulas.
Por otro lado, aunque de acuerdo con esta primera aproximación no es posible identificar la magnitud de los usuarios del Homeschooling o enseñantes en casa, si es posible determinar que en México existen condiciones para que los padres, que así lo deseen, puedan ir enseñando a sus hijos en casa, pero también es necesario reconocer que no existen los mecanismo mediante los cuales los padres cuenten con la orientación necesaria para realizar dicha tarea, además de que se requiere que cuenten con los espacios y mecanismos necesarios para ir validando de manera paulatina los avances formativos que van teniendo sus hijos.

No obstante lo anterior, reconocemos que es necesario seguir indagando sobre lo que es e implica el proceso formativo en casa -Homeschooling-, para lograr su comprensión y con ello estar en posibilidad de generar propuestas para dar respuesta desde estas alternativas educativas.

\section{Referencias}

Abraham, Sharon Dennise (2016). Experiencias y significados del Homeschooling: la voz de los padres de familia. Tesis de Licenciatura. México: Facultad de Filosofía y Letras. Universidad Nacional Autónoma de México. (UNAM). https://repositorio.unam.mx/contenidos/experiencias-ysignificados-del-homeschooling-en-mexico-la-voz-de-lospadres-de-familia$440200 ? \mathrm{c}=\mathrm{rY} 71 \mathrm{NV} \& \mathrm{~d}=$ false $\& \mathrm{q}=$ humanida $\mathrm{des} \& \mathrm{i}=1 \& \mathrm{v}=1 \& \mathrm{t}=\mathrm{s}$ earch_0\&as=0 [25 de octubre del 2019].

Alvarado Guzmán, Daniel Enrique; Montemayor Molina, Adriana Lucía y Rosales Vargas, Ana Goretti (2018). "La educación en casa (homeschooling) en familias cristianas del noreste de México". [En línea], enero-abril, 2018. Revista Axón. Disponible en: https://tyreditorial.com/pdf/Axon/1/6.pdf [20 de octubre del 2019].

Alvarado Moreno, Felipe y Cerón Martínez, Armando Ulises (2015). Currículum, Educación de Masas y el Modelo de la Homeschooling. Ponencia. Congreso Internacional de Educación y Currículum [En línea]. Recuperado de: http://posgradoeducacionuatx.org/pdf2015/B020.pdf $[12 \mathrm{de}$ noviembre del 2019].

AprendeconAlas.net Disponible en: https://www.aprendeconalas.com/

Castells, Manuel (2000) La era de la información. La sociedad red. Madrid: Alianza.

Clarenc, Claudio Ariel (2013). Analizamos 19 plataformas de eLearning: Investigación colaborativa sobre LMS. Grupo GEIPITE, Congreso Virtual Mundialde e-Learning.

Coll, César y Monereo Carles (Eds.) (2008) Psicologia de la Educación Virtual.Madrid: Morata.educazion.net. Disponible en línea en: https://educazion.net/info/

Hoeneisen, Alexandra (2014). La educación en casa: una modalidad educativa a considerar. Tesis de Licenciatura en Educación. Quito, Ecuador. Colegio de Ciencias Sociales y Humanidades. Universidad de San Francisco de Quito. [En línea]. Disponible en: http://repositorio.usfq.edu.ec/handle/23000/3971 [15 de noviembre del 2019].

Gutiérrez, Luis (2012). "Conectivismo como teoría de aprendizaje: conceptos, ideas y posibles limitaciones". Revista educación y 
tecnología. [En línea] No. 1. Chile. Disponible en línea en: https://dialnet.unirioja .es/servlet/articulo?codigo $=4169414 \quad[12$ de noviembre de 2019].

Instituto Hidalguense de Educación para Adultos (IHEA). Disponible en línea: http://ihea.hidalgo.gob.mx/

Instituto Nacional para la Educación de Adultos (INEA). Disponible en línea: http://www.inea.gob.mx/index.php

Matti School curriculum. http://www.matihomeschool.com/curriculumhomeschool-espanol/

MEVyTenLínea.http://mevytenlinea.inea.gob.mx/inicio/index.html

Mieles, María Dilia y García, María Cristina (2010). "Apuntes sobre socia lización infantil y construcción de identidad en ambientes multiculturales". [En línea]. Revista Latinoamericana de Ciencias Sociales, Niñez y Juventud, Vol. 8, No. 2, juliodiciembre 2010. Centro de Estudios Avanzados en Niñez y Juventud Alianza de la Universidad de Manizales y del CINDE. http://biblioteca.clacso.edu.ar/Colombia/alianza -cindeumz/20140320055527/art.MariaDiliaMieles.pdf $[25$ de noviembre del 2019].

Pineda, Angélica. (2016). El uso de Tecnologías de la Informa ción y la Comunicación en casa: una aproximación desde la Teoría Fundamentada. Tesis de Maestría en Informática Educativa. Chía Colombia. Centro de Tecnologías para la Academia. Universidad de la Sabana. Disponible en: https://intellectum.unisabana.edu.co/bitstream/handle/10818/27 687/Ang\%C3\%A9lica\%20Pineda \%20Franky\%20(Tesis).pdf?se quence $=1 \&$ isAllowed $=y \quad[30$ de noviembre del 2019].

Sánchez-Rodríguez, Karla (2016). Análisis descriptivo sobre el homeschooling en México. Tesis de Licenciatura en Pedagogía. México. Universidad Pedagógica Nacional. Unidad Ajusco. Disponible en línea en: http://200.23.113.51/pdf/32812.pdf [05 de diciembre del 2019].

Shayo, Conrad, Olfman, Lorne, Iriberri, Alicia \& Igbaria, Magid (2007). The Virtual Society: Its Driving Forces, Arrangements, Practices, and Implications, pp. 187-220. En: Gackenbach, Jayne (Ed). Psychology and the Internet. San Diego California: Elsevier. 